\title{
Spatiotemporal Dynamics of Feature-Based Attention Spread: Evidence from Combined Electroencephalographic and Magnetoencephalographic Recordings
}

\author{
Christian Michael Stoppel, ${ }^{1}$ Carsten Nicolas Boehler, ${ }^{2,3}$ Hendrik Strumpf, ${ }^{1}$ Ruth Marie Krebs, ${ }^{1,3}$ Hans-Jochen Heinze, ${ }^{1,2}$ \\ Jens-Max Hopf, ${ }^{1,2}$ and Mircea Ariel Schoenfeld ${ }^{1,2,4}$ \\ ${ }^{1}$ Department of Neurology and Center for Advanced Imaging, Otto-von-Guericke-University, 39120 Magdeburg, Germany, ${ }^{2}$ Leibniz-Institute for \\ Neurobiology, 39118 Magdeburg, Germany, ${ }^{3}$ Department of Experimental Psychology, Ghent University, 9000 Ghent, Belgium, and ${ }^{4}$ Kliniken Schmieder, \\ 78476 Allensbach, Germany
}

Attentional selection on the basis of nonspatial stimulus features induces a sensory gain enhancement by increasing the firing-rate of individual neurons tuned to the attended feature, while responses of neurons tuned to opposite feature-values are suppressed. Here we recorded event-related potentials (ERPs) and magnetic fields (ERMFs) in human observers to investigate the underlying neural correlates of feature-based attention at the population level. During the task subjects attended to a moving transparent surface presented in the left visual field, while task-irrelevant probe stimuli executing brief movements into varying directions were presented in the opposite visual field. ERP and ERMF amplitudes elicited by the unattended task-irrelevant probes were modulated as a function of the similarity between their movement direction and the task-relevant movement direction in the attended visual field. These activity modulations reflecting globally enhanced processing of the attended feature were observed to start not before 200 ms poststimulus and were localized to the motion-sensitive area hMT. The current results indicate that feature-based attention operates in a global manner but needs time to spread and provide strong support for the feature-similarity gain model.

\section{Introduction}

The capacity for perceptual analysis of incoming sensory information is limited within the human brain. Consequently, when multiple stimuli compete for representation, our visual system has to select which input should be preferentially processed at the expense of other information (Desimone and Duncan, 1995; Serences and Yantis, 2006). Psychophysical and neurophysiological evidence indicates that this selection can be accomplished by focusing attention to particular spatial locations (Posner, 1980; Heinze et al., 1994). Furthermore, attention may also be deployed to the nonspatial attributes of a stimulus, such as its color, shape, or motion (Hillyard and Münte, 1984; Corbetta et al., 1990; Britten et al., 1992), which results in increased activity within those cortical modules that are specialized in processing the respective features (O'Craven et al., 1997; Chawla et al., 1999; Schoenfeld et al., 2007).

Beyond this response enhancement for attended compared with unattended stimulus-features, pioneering studies by Treue and coworkers (Treue and Martínez Trujillo, 1999; Treue and

Received Jan. 31, 2012; revised April 28, 2012; accepted May 4, 2012.

Author contributions: C.M.S., C.N.B., J.-M.H., and M.A.S. designed research; C.M.S. and H.S. performed research; C.M.S., H.S., and M.A.S. analyzed data; C.M.S., C.N.B., R.M.K., H.-J.H., J.-M.H., and M.A.S. wrote the paper.

This work was supported by the following grants: Scho 1217/1-2 and SFB 779-A1 from the Deutsche Forschungsgemeinschaft (DFG) awarded to M.A.S.

Correspondence should be addressed to Christian Stoppel, Department of Neurology, Otto-von-GuerickeUniversity Magdeburg, Leipziger Strasse 44, 39120 Magdeburg, Germany. E-mail: christian.stoppel@med.ovgu.de. DOI:10.1523/JNEUROSCI.0439-12.2012

Copyright $\odot 2012$ the authors $\quad 0270-6474 / 12 / 329671-06 \$ 15.00 / 0$
Maunsell, 1999; Martinez-Trujillo and Treue, 2004) demonstrated that feature-based attention modifies the response profile of feature-selective neurons in a multiplicative manner. This "feature-similarity gain" mechanism operates by amplifying the firing-rate of neurons whose feature-preference closely matches the attended feature value (e.g., one specific motion direction of a stimulus), while the firing of neurons tuned to opposite feature-values (e.g., other motion directions) is suppressed. This multiplicative gain enhancement observed by singleneuron recordings in primates has been proposed to result in improved feature selectivity at the level of an integrated population response. Recently this assumption has been confirmed using fMRI in human observers (Kamitani and Tong, 2006; Stoppel et al., 2011), with stimuli that were presented within the focus of spatial attention.

In addition to these feature-based effects within the focus of attention, feature-selection has also been shown to modulate the firing-rate of neurons in an entirely location-independent manner. An enhanced response of neurons tuned to a specific feature could be observed although stimuli were presented outside of the neuron's receptive field (Treue and Martínez Trujillo, 1999; Martinez-Trujillo and Treue, 2004; Bichot et al., 2005). Recently, such spatially global feature-selective modulations have also been described at the population-level using fMRI (Saenz et al., 2002; Kamitani and Tong, 2006; Serences and Boynton, 2007) and recordings of steady-state visual evoked potentials in humans (Andersen et al., 2009, 2011). Nevertheless, little is known about the timing of this type of modulation. 
In the present study we simultaneously recorded electroencephalographic and magnetoencephalographic activity timelocked to the motion onset of a spatially unattended random-dot kinematogram to investigate the time course and the neural substrates of global feature-based attentional selection at the population level. Participants were required to attend to a moving transparent surface to perform in a motion discrimination task, while a second surface presented to the opposite visual field performed brief transient movements into varying directions. This design permitted us to quantify the magnitude and latency of event-related potentials (ERPs) and event-related magnetic fields (ERMFs) evoked by the unattended surface, in dependence of the similarity between its motion direction and that of the attended surface.

\section{Materials and Methods}

Subjects. Sixteen right-handed healthy subjects (mean age: $27.0 \pm 3.7 \mathrm{SD}$ years, 4 males), all with normal or corrected-to-normal visual acuity, participated as paid volunteers in the study. The local ethics committee of the Otto-vonGuericke University Magdeburg approved the experiment and all subjects gave written informed consent before participation.

Stimuli and experimental design. Stimuli were presented against a dark background $\left(0.5 \mathrm{~cd} / \mathrm{m}^{2}\right)$ within two square apertures $\left(4.2^{\circ} \times 4.2^{\circ}\right)$ centered $5.7^{\circ}$ to the left and right of a central fixation cross $\left(0.8^{\circ} \times 0.8^{\circ}\right.$; Fig. 1). Each aperture contained 100 randomly distributed isoluminant white dots (brightness $200 \mathrm{~cd} / \mathrm{m}^{2}$; dot size $0.08^{\circ}$ ). All dots within the left aperture moved either coherently upward (during even runs) or downward (during odd runs; velocity: $10 \%$ ) and were perceived as a transparent surface. The subjects' task was to attend to this surface and to make a speeded button-press response after detecting an accelerated movement of the attended surface (velocity: $22 \%$ for $300 \mathrm{~ms}$ ). Within the right aperture all dots remained stationary throughout the experiment except during probe trials in which all dots coherently performed a short displacement into one of the eight cardinal or ordinal directions (velocity: $10 \%$ for $200 \mathrm{~ms}$ ). These probe movements deviated from the motion direction of the attended surface by $0^{\circ}, 45^{\circ}, 90^{\circ}, 135^{\circ}$, or $180^{\circ}$ (Fig. 1 ) and were completely task-irrelevant. During target trials (accelerated movement of the attended surface in the left hemifield) the dots in the unattended visual field always remained stationary. Conversely, on probe trials (short displacement of the dots in the unattended aperture in the right hemifield) there was never an accelerated movement of the attended surface. Thus, probe and target trials always occurred independent of each other in a predefined pseudo-random sequence, and all trial types (targets and each of the probe types of different directionality) were presented equally often throughout the experiment. All trials (targets, as well as probes) were separated by an intertrial interval that varied randomly between 1250 and $1750 \mathrm{~ms}$ (mean $1500 \mathrm{~ms}$ ). The experiment was performed in seven runs of $385 \mathrm{~s}$ each with rest periods in-between. Each run consisted of 252 trials, resulting in a total of 294 trials per condition. Throughout the experiment subjects were instructed to keep accurate fixation, which was monitored by electro-oculogram (EOG; see below).

Data acquisition. ERPs and ERMFs were simultaneously recorded using a Magnes 3600 whole-head magnetoencephalographic (MEG) system with 248 magnetometer and 32 electroencephalographic (EEG) channels (4-D Neuroimaging/Biomagnetic Technologies Inc.). The signals were digitized at a rate of $508 \mathrm{~Hz}$ with an online bandpass of DC to $200 \mathrm{~Hz}$. The horizontal EOG was recorded using a bipolar montage with
2 electrodes behind the lateral orbital angles, whereas the vertical EOG was recorded from an electrode below the right orbital limb. Impedances were kept below $5 \mathrm{k} \Omega$ and a midline fronto-polar electrode served as ground. MEG signals were submitted to online and offline noise reduction (Robinson, 1989), and an artifact rejection was applied with peakto-peak limits of $2-4 \mathrm{pT}$ for the MEG and $80-200 \mu \mathrm{V}$ for the EOG signal (thresholds were adjusted individually for each subject, but were constant over all experimental conditions). Individual head shapes were coregistered with the sensor coordinate system by digitizing (Polhemus 3Space Fastrak system) skull landmarks (nasion, left, and right preauricular points) and determining their locations relative to sensor and electrode positions using signals from 5 spatially distributed coils attached to the subjects' heads. To account for differences in head positions relative to the MEG sensors, the individual subjects' MEG data were aligned using the following procedures. First a lead field was computed (using Curry 6.01 Compumedics Neuroscan) for each subject using the subjects individual sensor-configuration. Then an inverse solution for this lead field was computed (using generalized least-squares methods) without applying any sensor- or dipol-weighting. Finally regularization by a modeling-parameter lambda was performed to prevent from ghostactivity in source-space. Lambda was chosen to make a compromise between large full-width-half-maximum-values of point-spreadfunction and noise-increment by ghost-activity. The result was an inverse solution that is independent from the measured sensor-data. In a second step a forward solution was computed for a reference sensor grid of 248 sensors. The individual subject's data, now in the reference sensor grid, were averaged together to obtain the grand average.

Data analysis. Separate ERP and ERMF average waveforms were computed time-locked to the motion onset for each of the 5 probe conditions. Attention effects were quantified in these average waveforms as mean amplitude measures within latency intervals of $110-210$ and $210-310 \mathrm{~ms}$ poststimulus onset (with respect to a $200 \mathrm{~ms}$ prestimulus baseline) at the sensor/electrode sites showing the largest amplitudes. Statistical analysis of the data was performed using within-subjects repeated ANOVAs (Greenhouse-Geisser correction was applied when necessary). To determine the time of onset of the attention effects, amplitude measures were taken over successive $10 \mathrm{~ms}$ intervals and tested for significant differences 


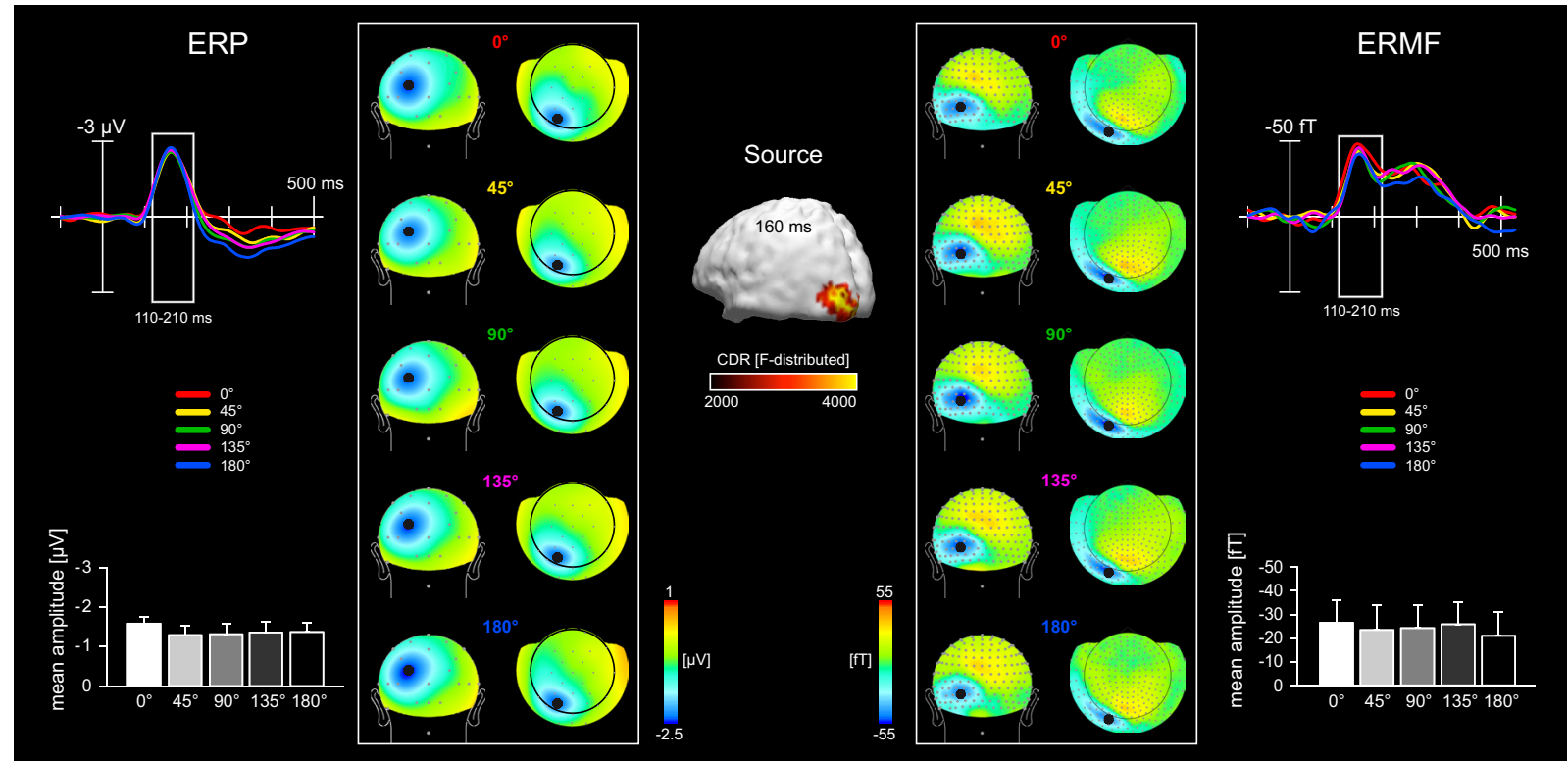

Figure 2. No global feature-based attention effects in the time range of the $\mathrm{N} 1$ component ( $110-210 \mathrm{~ms}$ poststimulus onset). Time courses and mean amplitudes of the probe-related ERP (left column) and ERMF (right column) responses in the time range between 110 and $210 \mathrm{~ms}$ after onset of the probe stimuli. Recording sites are indicated as black dots within the field distribution maps. The magnitude of ERP and ERMF amplitudes shows no dependency on the motion direction of the attended surface. A minimum in the ERP field distribution can been seen over left parieto-occipital electrode sites (left topography maps), accompanied by an efflux-influx distribution of the ERMFs, which is located over left occipital sensors (right topography maps). The estimated current source density distribution $160 \mathrm{~ms}$ poststimulus onset shows one maximum located in left posterior lateral extrastriate cortex.

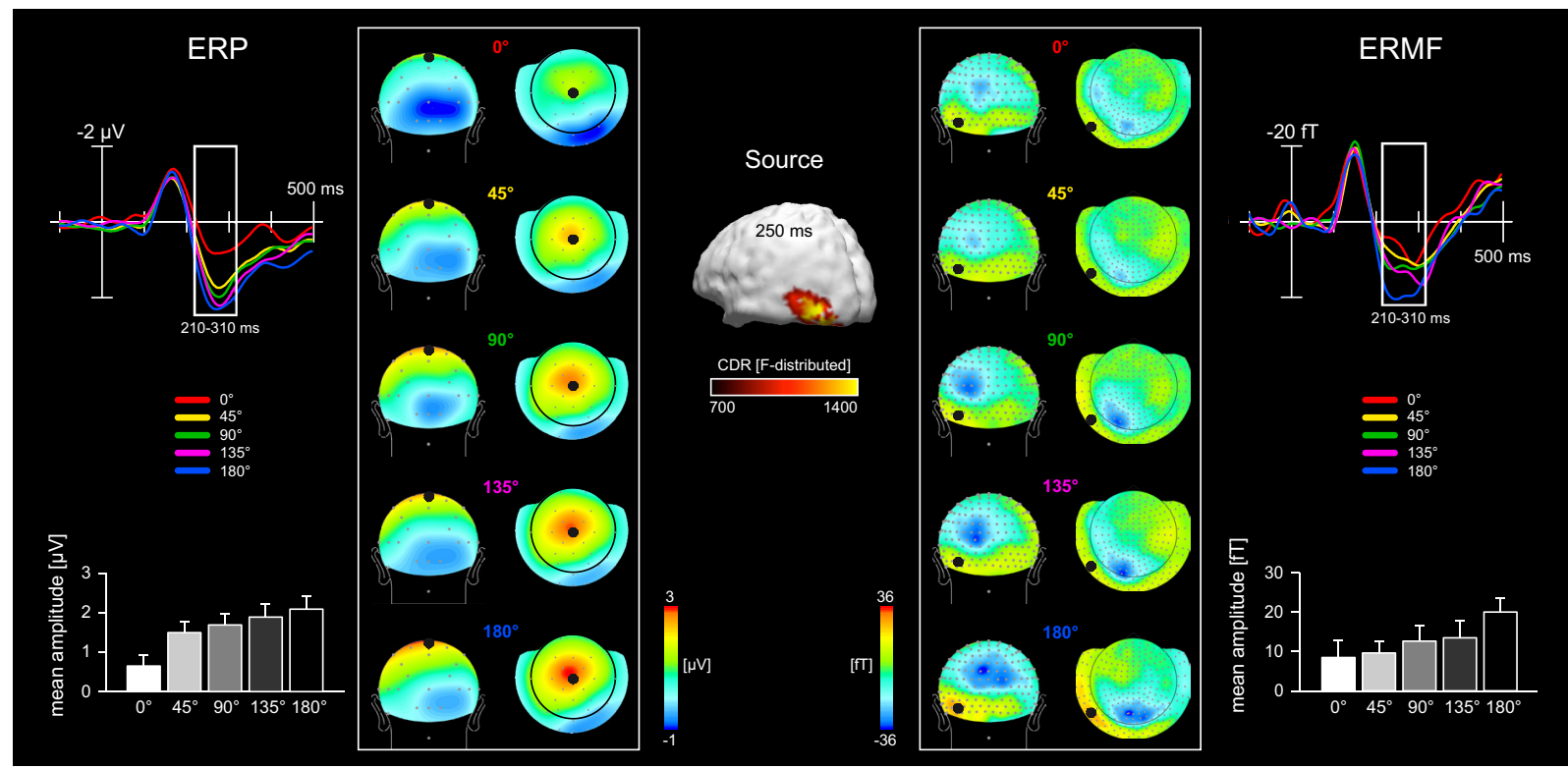

Figure 3. Global feature-based attentional modulations between 210 and $310 \mathrm{~ms}$ poststimulus onset. Time courses and mean amplitudes ( $210-310 \mathrm{~ms}$ poststimulus onset) of the probe-related ERP (left column) and ERMF (right column) responses. Recording sites are indicated as black dots within the field distribution maps. Note that the magnitude of the ERP and ERMF amplitudes parametrically depends on the deviation of the probes' motion direction from the direction of the attended surface (indicated in degrees of visual angle by which the probes deviated from the attended motion direction). The topographical field distributions (averaged over the time range 210-310 ms) show a maximal positivity over midline central electrode sites for the ERPs (left topography maps) and an efflux-influx field transition located over left occipitotemporal sensors for the ERMFs (right topography maps). The estimated current source density distribution $250 \mathrm{~ms}$ poststimulus onset (displayed in the middle of the figure) shows one maximum located in the left middle occipitotemporal cortex.

between conditions with a criterion of $p<0.05$. The earliest significant interval followed by 5 (or more) successive significant intervals was taken as the onset latency (Guthrie and Buchwald, 1991; Schoenfeld et al., 2003, 2007).

Source localization. For source localization, current source density estimates were computed by means of standardized low-resolution electromagnetic tomography (sLORETA; Pascual-Marqui, 2002) as implemented in the neuroimaging software Curry 6.01 (Compumedics Neuroscan). The sLORETA represents an extension of the minimum norm least square method (Hämäläinen and Ilmoniemi, 1994; Fuchs et al., 1999), where current estimates at each source location are weighted by their measurement error, yielding a pseudo- $F$-value distribution of currents over the cortical surface, called source density estimates (SDEs). All source localization results provided in Figures 2 and 3 represent such SDEs. Since the distribution of magnetic fields measured by MEG is oriented perpendicularly to the concurrent voltage field distribution assessed by EEG, the surface topographies of both fields elicited by a given dipolar source display a nearly orthogonal surface topography. There- 
fore, both the ERP and ERMF distributions were concurrently fit by sLORETA to obtain maximal localization power (Fuchs et al., 1998; Schoenfeld et al., 2003). One prerequisite for the concomitant use of both the ERP and ERMF field distributions is that the conductivities of the volume conductor model are matched for the EEG and MEG recordings. Therefore, a conductivity factor was determined to scale the EEG relative to the MEG data based on a tangential dipole evoked by tactile stimulation of the index finger by an air puff at 30-40 ms latency (Fuchs et al., 1998). This conductivity factor could be reliably approximated to a value of 0.8 and was used for estimation of the source localization on the average data across all subjects. All inverse computations were constrained by realistic anatomical models of volume conductor and source compartment derived by 3-dimensional surface reconstructions of the head, CSF space, and cortical surface, respectively (boundary element method; Hämäläinen and Sarvas, 1989). The anatomical basis for the source analysis was the Montreal Neurological Institute brain (average of $152 \mathrm{~T} 1$-weighted stereotaxic volumes).

\section{Results}

\section{Behavioral results}

Subject were accurate at detecting the faster moving targets, with a mean hit rate of $95.5 \%$ (SD: $\pm 4.1 \%$ ) and a false alarm rate of $2.9 \%$ (SD: $\pm 2.6 \%$ ). Mean reaction times ranged from 414 to 501 ms (mean \pm SD: $458 \pm 31 \mathrm{~ms}$ ).

\section{ERP/ ERMF results}

The effects of feature-based attention on direction-selective neural activity were assessed, by comparing the ERP/ERMF waveforms elicited by the different probe stimuli. The ERP/ERMF amplitudes within the time-range of the N1-component (110$210 \mathrm{~ms}$ ) were not significantly modulated by the similarity between the motion direction of the attended surface and that of the moving probe stimuli (ERPs, $F_{(4,60)}=0.99, p>0.4$, Fig. 2, left column; ERMFs, $F_{(4,60)}=0.97 ; p>0.4$, Fig. 2 , right column). The topographical field distributions for all probe conditions showed a maximal negativity over left parieto-occipital electrodes in the ERPs (Fig. 2, left topography maps) and one efflux-influx field configuration located over left occipital sensors for the ERMFs with only minimal variations in amplitude (Fig. 2, right topography maps). The corresponding current source distribution within the $\mathrm{N} 1$ time-range (at $160 \mathrm{~ms}$ poststimulus onset) revealed source-activity estimates located in the left posterior lateral extrastriate cortex (Talairach coordinates: -35/-93/7) most likely corresponding to visual areas V2 and V3.

In contrast, the ERP/ERMF amplitudes in the time-range between 210 and $310 \mathrm{~ms}$ were modulated as a function of the similarity between the motion direction of the probe and the direction of the attended surface, with more negative ERP $\left(F_{(4,60)}=10.77 ; p<0.0001\right.$; Fig. 3, left column $)$ and ERMF amplitudes $\left(F_{(4,60)}=3.57 ; p<0.05\right.$; Fig. 3, right column $)$ for probe stimuli matching more closely the attended direction. Note that the magnitude of the ERP and ERMF amplitudes between 210 and $310 \mathrm{~ms}$ decreases as a function of the deviation of the probes' motion direction from that of the attended surface. Statistical comparison in successive $10 \mathrm{~ms}$ epochs indicated that these differences between probe conditions became significant at $\sim 200$ ms post-probe. In the subsequent interval between 210 and 310 $\mathrm{ms}$, the ERP field distribution map showed a maximal positivity over midline central electrode sites (Fig. 3, left topography maps), accompanied by an efflux-influx field configuration located over left occipitotemporal sensors for the ERMFs (Fig. 3, right topography maps). The source analysis revealed estimates of activity (sLORETA estimates, see Materials and Methods) located in left middle occipitotemporal cortex (Talairach coordinates: $-46 /$ $-77 /-1)$, most likely corresponding to region V5/hMT.

\section{Discussion}

The present study used simultaneous recordings of ERPs and ERMFs in human observers to investigate the spatiotemporal correlates of feature-based attentional selection at the neural population level. During the task subjects attended to a moving transparent surface in the left visual field and performed a motion discrimination task, while a task-irrelevant second surface located in the opposite visual field moved into different directions. This experimental design permitted to quantify the magnitude of ERPs and ERMFs evoked by the unattended surface while systematically varying the similarity between its motion direction and that of the attended surface. Our current results demonstrate a parametric feature-based attentional modulation of ERP and ERMF amplitudes, as a function of the similarity between the motion directions of the spatially attended and unattended stimuli. The time courses of the ERP and ERMF waveforms indicate that this attentional enhancement starts not before $200 \mathrm{~ms}$ poststimulus onset and originates from left middle occipitotemporal cortex, most likely corresponding to area V5/hMT. These findings provide strong support for the feature-similarity gain model by demonstrating that feature-based attention parametrically modulates direction-selective population activity within V5/ hMT in a global manner. The timing indicates that the global spread of attention toward spatially unattended locations does not occur immediately after the selection of the attended feature pointing out to the time-consuming nature of this process.

The observed feature-based modulations were localized to the lateral middle occipitotemporal cortex, which corresponds well to area V5/hMT. This region, considered to be the homolog of the well described MT region in non-human primates, is specialized for the processing of motion information (Zeki et al., 1991; Ahlfors et al., 1999). Beyond purely sensory-driven effects, activity within this region can be markedly affected by attention. For example, V5/MT neurons increase their firing rate to attended compared with unattended motion stimuli (Cook and Maunsell, 2002, 2004), which is consistent with findings in human subjects (Corbetta et al., 1990, 1991; O'Craven et al., 1997; Schoenfeld et al., 2007). In addition to this global response enhancement, recent observations indicated that V5/MT activity could also be affected in a direction-selective manner. Feature-based attention modifies the response profile of direction-selective neurons within V5/MT multiplicatively: neurons whose feature preference closely match an attended motion direction increase their firing rate, while the firing of neurons tuned to opposite directions is suppressed (Treue and Martínez Trujillo, 1999; MartinezTrujillo and Treue, 2004). As a consequence of these findings the 'feature-similarity gain model' has been formulated. This model posits that an individual neuron's response depends on the feature similarity between a current behaviorally relevant target and the feature preference of that neuron, resulting in an improved selectivity for the attended feature at the population level. Recently this hypothesis has also been confirmed using fMRI experiments in human observers with stimuli that were presented within the focus of spatial attention (Kamitani and Tong, 2006; Stoppel et al., 2011). Another fMRI study indicated that featurebased attention might also operate in a spatially global manner, i.e., for stimuli occurring at spatially unattended locations (Saenz et al., 2002). However, this study only compared stimuli that moved into the attended versus opposed to the attended direction. More importantly, due to the nature of the fMRI technique no inferences could be made on the timing. The current results extend these findings (Saenz et al., 2002) by demonstrating that 
electromagnetic population activity (i.e., ERP/ERMF amplitudes) to moving stimuli presented at unattended locations scales parametrically in dependence of their feature-similarity with respect to the attended stimulus.

Although fMRI studies provided a detailed picture on the anatomical structures modulated by feature-based selection, their temporal resolution is too limited to reveal the timing of the underlying attentional modulations. Fine-grained information about the time course of feature-based selection has therefore been determined primarily based on data from noninvasive EEG/ MEG recordings in humans. By this means, previous studies indicated that the selection of task-relevant features is initiated in the time range of the N1-component, i.e., between 100 and 180 ms poststimulus onset (Harter and Aine, 1984; Kenemans et al., 1993; Motter, 1994; Anllo-Vento and Hillyard, 1996; Smid et al., 1999; Torriente et al., 1999; Kenemans et al., 2000; Martínez et al., 2001; Beer and Röder, 2004, 2005; Schoenfeld et al., 2007), which in most cases is reflected by a broad negativity over centroposterior electrodes in the ERP (the so-called selection-negativity; Harter and Aine, 1984; Hillyard and Anllo-Vento, 1998). A common feature of these studies was that the stimulus eliciting the neurophysiological response was located in the attended part of the space.

Thus, the feature-based selection occurred at the spatially attended location. In the present study we observed an enhanced negativity over centro-posterior electrodes in the EEG, whose magnitude parametrically depended on the similarity between the motion directions of the attended and the unattended surfaces (Fig. 3). The corresponding modulations were also observed in the simultaneously recorded ERMF over occipitotemporal sensors. Importantly, the onset latency of these modulations was later $(\approx 200 \mathrm{~ms}$ ) than previously reported (Hillyard and Münte, 1984; Anllo-Vento and Hillyard, 1996; Karayanidis and Michie, 1996; Lange et al., 1998; Schoenfeld et al., 2007). Given that in the present experiment the motion probes were located in the unattended visual field, this latency difference is likely to reflect the temporal costs underlying the spread of feature-selective modulations toward spatially unattended locations.

However, there are also other explanations that might account for the observed latency difference. One possibility would be that the delay results mainly from time costs related to involuntary attention capture processes triggered by the task-irrelevant stimuli (Egeth and Yantis, 1997). On the other hand the initiation of an involuntary attention shift towards the task-irrelevant stimulus could be regarded as an integral process of the featureselective attention spread that might be used to determine the most useful direction of propagation.

A second possibility would be that the observed latency difference rather reflects a delay in the general processing of the taskirrelevant stimulus than the time costs of the feature-based attentional spread. However, the processing of task-relevant stimuli within the same feature value (e.g., one color from another) at an attended location is typically enhanced at $\sim 100-110$ ms poststimulus (Anllo-Vento et al., 1998). The processing of a task-irrelevant feature at the attended location is typically delayed by $\sim 50 \mathrm{~ms}$ (Schoenfeld et al., 2003). A general delay due to the task irrelevance per se would therefore be expected to be reflected in the electrophysiological recordings $\sim 150-160 \mathrm{~ms}$ poststimulus, which is different from the timing observed in the present study ( $\sim 200 \mathrm{~ms})$. Task relevance is certainly a key factor with regard to the timing differences, but is unlikely to cause the entire delay observed.

Together with other findings from the literature, the current results point to a more general framework of the temporal dy- namics of attentional spreading. Within an attended spatial location feature selection can be very fast $(\sim 100 \mathrm{~ms}$ poststimulus onset). This is especially the case when an entire featuredimension can be selected from another one (e.g., attending a stimulus' motion vs its color; Schoenfeld et al., 2007). Additional time costs are observed when attentional selection operates within a single feature-dimension (e.g., selecting one particular motion direction from another; Hillyard and Münte, 1984; Anllo-Vento and Hillyard, 1996; Karayanidis and Michie, 1996; Lange et al., 1998). Depending on the stimuli and task used, attentional selection occurs $\sim 110-160 \mathrm{~ms}$ poststimulus. In the current experiment the probe was located in the spatially unattended visual field. In this case the attentional selection occurred $\sim 50 \mathrm{~ms}$ later ( $\sim 200 \mathrm{~ms}$ poststimulus onset) indicating that the spread of attention across spatial locations takes $\sim 50 \mathrm{~ms}$ of time. Importantly, not only the spread of attention over space takes time. Studies on object-based attention could show that the attentional spread from an attended to an unattended feature of that same object took $\sim 40-50 \mathrm{~ms}$. In this case both features were present at the same spatial location that was attended (Schoenfeld et al., 2003). A recent study showed that attention not only spreads across the same objects but also to other objects at spatially unattended locations if they share a task-irrelevant feature of an attended object (Boehler et al., 2011). In this case the attentional boost occurs even later, at $\sim 270 \mathrm{~ms}$ poststimulus. This suggests that the temporal costs for the attention spread from the task-relevant to the task-irrelevant object feature sum up to the cost for spreading from attended to unattended locations. Around $150 \mathrm{~ms}$ are needed for the selection of the task-relevant feature of a spatially attended object (Hillyard and Münte, 1984; Anllo-Vento and Hillyard, 1996; Karayanidis and Michie, 1996; Lange et al., 1998). Another 50-60 ms are needed for spreading to the task-irrelevant feature of the same object (Schoenfeld et al., 2003). The following spread toward an unattended spatial location would take another 50-60 ms (result of the current study). The resulting total time would be in the range between 250 and $270 \mathrm{~ms}$, which is well in line with the aforementioned findings (Boehler et al., 2011).

In conclusion, the current results show that feature-based attention is associated with a global processing enhancement of the attended feature (Treue and Martínez Trujillo, 1999; Saenz et al., 2002; Kamitani and Tong, 2006). Neural responses elicited by spatially unattended task-irrelevant probes were modulated as a function of the degree of similarity between their movement direction and the task-relevant movement direction in the attended visual field. This provides strong support for the "feature similarity gain model" at the level of integrated population responses (Treue and Martínez Trujillo, 1999; Stoppel et al., 2011). Importantly, the global spread of feature-based attention does not occur suddenly following feature selection but rather appears to be a dynamic time-consuming process.

\section{References}

Ahlfors SP, Simpson GV, Dale AM, Belliveau JW, Liu AK, Korvenoja A Virtanen J, Huotilainen M, Tootell RB, Aronen HJ, Ilmoniemi RJ (1999) Spatiotemporal activity of a cortical network for processing visual motion revealed by MEG and fMRI. J Neurophysiol 82:2545-2555.

Andersen SK, Muller MM, Hillyard SA (2009) Color-selective attention need not be mediated by spatial attention. J Vis 9:2.1-2.7.

Andersen SK, Fuchs S, Müller MM (2011) Effects of feature-selective and spatial attention at different stages of visual processing. J Cogn Neurosci 23:238-246.

Anllo-Vento L, Hillyard SA (1996) Selective attention to the color and direction of moving stimuli: electrophysiological correlates of hierarchical feature selection. Percept Psychophys 58:191-206. 
Anllo-Vento L, Luck SJ, Hillyard SA (1998) Spatio-temporal dynamics of attention to color: evidence from human electrophysiology. Hum Brain Mapp 6:216-238.

Beer AL, Röder B (2004) Attention to motion enhances processing of both visual and auditory stimuli: an event-related potential study. Brain Res Cogn Brain Res 18:205-225.

Beer AL, Röder B (2005) Attending to visual or auditory motion affects perception within and across modalities: an event-related potential study. Eur J Neurosci 21:1116-1130.

Bichot NP, Rossi AF, Desimone R (2005) Parallel and serial neural mechanisms for visual search in macaque area V4. Science 308:529-534.

Boehler CN, Schoenfeld MA, Heinze HJ, Hopf JM (2011) Object-based selection of irrelevant features is not confined to the attended object. J Cogn Neurosci 23:2231-2239.

Britten KH, Shadlen MN, Newsome WT, Movshon JA (1992) The analysis of visual motion: a comparison of neuronal and psychophysical performance. J Neurosci 12:4745-4765.

Chawla D, Rees G, Friston KJ (1999) The physiological basis of attentional modulation in extrastriate visual areas. Nat Neurosci 2:671-676.

Cook EP, Maunsell JH (2002) Attentional modulation of behavioral performance and neuronal responses in middle temporal and ventral intraparietal areas of macaque monkey. J Neurosci 22:1994-2004.

Cook EP, Maunsell JH (2004) Attentional modulation of motion integration of individual neurons in the middle temporal visual area. J Neurosci 24:7964-7977.

Corbetta M, Miezin FM, Dobmeyer S, Shulman GL, Petersen SE (1990) Attentional modulation of neural processing of shape, color, and velocity in humans. Science 248:1556-1559.

Corbetta M, Miezin FM, Dobmeyer S, Shulman GL, Petersen SE (1991) Selective and divided attention during visual discriminations of shape, color, and speed: functional anatomy by positron emission tomography. J Neurosci 11:2383-2402.

Desimone R, Duncan J (1995) Neural mechanisms of selective visual attention. Annu Rev Neurosci 18:193-222.

Egeth HE, Yantis S (1997) Visual attention: control, representation, and time course. Annu Rev Psychol 48:269-297.

Fuchs M, Wagner M, Wischmann HA, Köhler T, Theissen A, Drenckhahn R, Buchner H (1998) Improving source reconstructions by combining bioelectric and biomagnetic data. Electroencephalogr Clin Neurophysiol 107:93-111.

Fuchs M, Wagner M, Köhler T, Wischmann HA (1999) Linear and nonlinear current density reconstructions. J Clin Neurophysiol 16:267-295.

Guthrie D, Buchwald JS (1991) Significance testing of difference potentials. Psychophysiology 28:240-244.

Hämäläinen MS, Ilmoniemi RJ (1994) Interpreting magnetic fields of the brain: minimum norm estimates. Med Biol Eng Comput 32:35-42.

Hämäläinen MS, Sarvas J (1989) Realistic conductivity geometry model of the human head for interpretation of neuromagnetic data. IEEE Trans Biomed Eng 36:165-171.

Harter MR, Aine C (1984) Brain mechanisms of visual selective attention. In: Varieties of attention (Parasuraman R, Davies DR, eds), pp 293-321. New York: Academic.

Heinze HJ, Mangun GR, Burchert W, Hinrichs H, Scholz M, Münte TF, Gös A, Scherg M, Johannes S, Hundeshagen H, Gazzaniga MS, Hillyard SA (1994) Combined spatial and temporal imaging of brain activity during visual selective attention in humans. Nature 372:543-546.

Hillyard SA, Anllo-Vento L (1998) Event-related brain potentials in the study of visual selective attention. Proc Natl Acad Sci U S A 95:781-787.

Hillyard SA, Münte TF (1984) Selective attention to color and location: an analysis with event-related brain potentials. Percept Psychophys 36:185-198.

Kamitani Y, Tong F (2006) Decoding seen and attended motion directions from activity in the human visual cortex. Curr Biol 16:1096-1102.
Karayanidis F, Michie PT (1996) Frontal processing negativity in a visual selective attention task. Electroencephalogr Clin Neurophysiol 99:38-56.

Kenemans JL, Kok A, Smulders FT (1993) Event-related potentials to conjunctions of spatial frequency and orientation as a function of stimulus parameters and response requirements. Electroencephalogr Clin Neurophysiol 88:51-63.

Kenemans JL, Baas JM, Mangun GR, Lijffijt M, Verbaten MN (2000) On the processing of spatial frequencies as revealed by evoked-potential source modeling. Clin Neurophysiol 111:1113-1123.

Lange JJ, Wijers AA, Mulder LJ, Mulder G (1998) Color selection and location selection in ERPs: differences, similarities and 'neural specificity'. Biol Psychol 48:153-182.

Martínez A, Di Russo F, Anllo-Vento L, Sereno MI, Buxton RB, Hillyard SA (2001) Putting spatial attention on the map: timing and localization of stimulus selection processes in striate and extrastriate visual areas. Vision Res 41:1437-1457.

Martinez-Trujillo JC, Treue S (2004) Feature-based attention increases the selectivity of population responses in primate visual cortex. Curr Biol 14:744-751.

Motter BC (1994) Neural correlates of attentive selection for color or luminance in extrastriate area V4. J Neurosci 14:2178-2189.

O'Craven KM, Rosen BR, Kwong KK, Treisman A, Savoy RL (1997) Voluntary attention modulates fMRI activity in human MT-MST. Neuron 18:591-598.

Pascual-Marqui RD (2002) Standardized low-resolution brain electromagnetic tomography (sLORETA): technical details. Methods Find Exp Clin Pharmacol 24 [Suppl D]:5-12.

Posner MI (1980) Orienting of attention. Q J Exp Psychol 32:3-25.

Robinson SE (1989) Environmental noise cancellation for biomagnetic measurements. In: Advances in biomagnetism. (Williamson SJ, Hoke M, Stroink G, Kotani M, eds), pp 721-724. New York: Plenum.

Saenz M, Buracas GT, Boynton GM (2002) Global effects of feature-based attention in human visual cortex. Nat Neurosci 5:631-632.

Schoenfeld MA, Tempelmann C, Martinez A, Hopf JM, Sattler C, Heinze HJ, Hillyard SA (2003) Dynamics of feature binding during object-selective attention. Proc Natl Acad Sci U S A 100:11806-11811.

Schoenfeld MA, Hopf JM, Martinez A, Mai HM, Sattler C, Gasde A, Heinze HJ, Hillyard SA (2007) Spatio-temporal analysis of feature-based attention. Cereb Cortex 17:2468-2477.

Serences JT, Boynton GM (2007) Feature-based attentional modulations in the absence of direct visual stimulation. Neuron 55:301-312.

Serences JT, Yantis S (2006) Selective visual attention and perceptual coherence. Trends Cogn Sci 10:38-45.

Smid HG, Jakob A, Heinze HJ (1999) An event-related brain potential study of visual selective attention to conjunctions of color and shape. Psychophysiology 36:264-279.

Stoppel CM, Boehler CN, Strumpf H, Heinze HJ, Noesselt T, Hopf JM, Schoenfeld MA (2011) Feature-based attention modulates directionselective hemodynamic activity within human MT. Hum Brain Mapp 32:2183-2192.

Torriente I, Valdes-Sosa M, Ramirez D, Bobes MA (1999) Visual evoked potentials related to motion-onset are modulated by attention. Vision Res 39:4122-4139.

Treue S, Martínez Trujillo JC (1999) Feature-based attention influences motion processing gain in macaque visual cortex. Nature 399:575-579.

Treue S, Maunsell JH (1999) Effects of attention on the processing of motion in macaque middle temporal and medial superior temporal visual cortical areas. J Neurosci 19:7591-7602.

Zeki S, Watson JD, Lueck CJ, Friston KJ, Kennard C, Frackowiak RS (1991) A direct demonstration of functional specialization in human visual cortex. J Neurosci 11:641-649. 